\title{
Mitochondrial microsatellite instability in patients with metastatic colorectal cancer
}

\author{
S. Venderbosch • S. van Vliet • M. H. C. Craenmehr • \\ F. Simmer • A. F. J. de Haan • C. J. A. Punt • \\ M. Koopman • I. D. Nagtegaal
}

Received: 22 October 2014 / Accepted: 2 February 2015 / Published online: 20 February 2015

(C) The Author(s) 2015. This article is published with open access at Springerlink.com

\begin{abstract}
Mitochondrial microsatellite instability (mtMSI), a change in length in mtDNA microsatellite sequences between normal and tumor tissue, has been described as a frequent occurrence in colorectal cancer (CRC). We evaluated the prevalence and prognostic value of mtMSI and its relation to nuclear microsatellite instability (MSI) in patients with metastatic CRC (mCRC). At six loci (D310, D514, D16184, ND1, ND5, and COX1), the mitochondrial DNA sequence was analyzed in normal and tumor tissue, and the mtMSI status was determined. We evaluated the prevalence and outcome in terms of overall survival (OS) in $83 \mathrm{CRC}$ patients with a MSI tumor (including 39 patients with Lynch syndrome) and in 99 mCRC patients with a microsatellite stable (MSS) tumor. A meta-analysis was performed to compare our findings with existing data. mtMSI at the D-loop region was found in $54.4 \%$ (99 out of 182) of all patients. Prevalence of mtMSI
\end{abstract}

Electronic supplementary material The online version of this article (doi:10.1007/s00428-015-1733-8) contains supplementary material, which is available to authorized users.

S. Venderbosch · S. van Vliet • M. H. C. Craenmehr · F. Simmer • I. D. Nagtegaal $(\square)$

Department of Pathology, Radboud University Medical Center, PO Box 9101, 6500 HB Nijmegen, The Netherlands

e-mail: Iris.Nagtegaal@radboudumc.nl

A. F. J. de Haan

Department for Health Evidence, Section Biostatistics, Radboud

University Medical Center, Nijmegen, The Netherlands

S. Venderbosch $\cdot$ C. J. A. Punt

Department of Medical Oncology, Academic Medical Centre,

University of Amsterdam, Amsterdam, The Netherlands

M. Koopman

Department of Medical Oncology, University Medical Centre Utrecht, Utrecht, The Netherlands was most pronounced at the D310 locus (50.5\%). Prevalence of mtMSI at the D-loop region was not different among patients with MSI compared to MSS tumors. There was no effect of mtMSI on prognosis in patients with MSI or MSS tumors. Prevalence of mtMSI was high in mCRC patients with both MSI and MSS tumors, but there was no correlation with prognosis. mtMSI was particularly present at the D310 locus.

Keywords Metastatic colorectal cancer $\cdot$ mtMSI

\section{Introduction}

Colorectal cancer (CRC) carcinogenesis is a multistep process involving the accumulation of genetic changes. $\mathrm{CRC}$ research is largely focused at activation of oncogenes, inactivation of tumor suppressor genes, and defects of mismatch repair genes in nuclear DNA. However, there are still many aspects that cannot be explained by these genetic changes.

More than half a century ago, Otto Warburg was the first to describe that mitochondria may play a role in carcinogenesis [47]. A mitochondrion is a membrane-enclosed intracellular organelle present in many copies per cell, which contains its own genetic system for replication, transcription, and translation [44]. Mitochondria generate most of the cell's supply of adenosine triphosphate (ATP), the major energy source of the cell, and are therefore involved in cell signaling, cellular differentiation, apoptosis, and control of the cell cycle and cell growth.

The mitochondrial DNA is a 16,569 -base pairs, doublestranded, circular DNA composed of genes and a noncoding region, the displacement loop (D-loop), which contains essential transcription and replication elements [30,45]. The genes 
encode for both a small (12S) and a large (16S) ribosomal RNA gene, 22 transfer RNAs and 13 proteins.

The genetics of mitochondria differ from genetics of the nuclear genome in 3 major characteristics: maternal inheritance, heteroplasmy, and mitotic segregation [8]. Initially, it was assumed that mitochondrial DNA is homoplasmic in normal cells, that is, all of the mitochondrial DNA copies are identical not only in an individual cell but also among cells. However, new sequencing techniques revealed that heteroplasmy is not restricted to cancer cells but can also be present in normal tissue [15].

It has been reported that mitochondrial DNA is more susceptible to mutations than nuclear DNA, due to the lack of histones and chromatin structure, paucity of introns, inefficient mitochondrial DNA repair mechanisms and a higher exposure to reactive oxygen species (ROS) produced by ATP synthesis $[33,41]$. Several hotspots of mitochondrial DNA mutations have been described in tumor tissue. A major target was found at the D310 sequence $\left(\mathrm{C}_{\mathrm{n}} \mathrm{TC}_{6}\right)$ in the non-coding D-loop [37]. In CRC, somatic mutations were also found at the D514 and D16184 sequence in the non-coding D-loop, rRNA genes, NADH dehydrogenase subunits (ND1, ND4L, and ND5), cytochrome $b$, and cytochrome oxidase subunits (COX1, COX2, and COX3). The majority of mutations were nucleotide substitutions or single base pair insertions. However, only few studies have sequenced the entire mitochondrial genome $[15,23]$; most studies focused only on a few regions or exclusively at the Dloop region in CRC [1, 2, 9, 16, 17, 19, 40, 46]. Data about mitochondrial DNA mutations are therefore scarce and inconsistent.

Mitochondrial microsatellite instability (mtMSI) has been defined as change in length in short base repetitive sequences of mitochondrial DNA (mtDNA) between normal and tumor tissue. mtMSI has been described as a frequent occurrence in human cancers $[4,5]$ and also in CRC $[12,14,35]$. Several studies have evaluated the relation of nuclear MSI with mtMSI, but results are inconclusive. The association between mtMSI and other clinical factors was also analyzed. In stage III CRC cancers, the presence of mtMSI at the D-loop is associated with poor prognosis and resistance to fluorouracilbased adjuvant chemotherapy [27].

To date, no data are available regarding the prevalence or prognostic value of mtMSI in metastatic CRC (mCRC) patients. We hypothesize that the presence of mtMSI might confer a worse prognosis in $\mathrm{mCRC}$, as seen in stage III CRC. We therefore assessed the role of mtMSI in respect to prevalence and outcome in patients with mCRC with either MSI or microsatellite stable (MSS) tumors.

\section{Materials and methods}

\section{Clinical samples}

Data were derived from mCRC patients included in two large phase III studies: CAIRO (Clinicaltrials.gov NCT00312000) and CAIRO2 (Clinicaltrials.gov NCT00208546), of which the results have been published previously [21, 42]. Collection of formalin-fixed paraffin-embedded (FFPE) material of the primary tumor was part of the initial protocol in both studies. To check that the normal colon tissue of CRC patients has a constant sequence pattern, we selected 10 patients from our hospital database for which we could obtain normal tissue from proximal and distal locations relative to the tumor.

In order to analyze the prevalence and relation between nuclear MSI and mtMSI, we selected $44 \mathrm{mCRC}$ patients with MSI tumors from the CAIRO studies (CAIRO [22], $n=19$, and CAIRO2 [42], $n=25$ ) and 39 CRC patients with a known Lynch syndrome from our own database. Furthermore, we selected $56 \mathrm{mCRC}$ patients with MSS tumors treated in the CAIRO study who were matched for known prognostic factors (the 'test group'); all patients that were treated with firstline capecitabine monotherapy for at least 3 cycles had a resection of the primary tumor, WHO performance score 0 , normal serum lactate dehydrogenase (LDH) concentrations, localization of the primary tumor in colon or rectosigmoid and had not received prior adjuvant chemotherapy. In order to validate the results found in the test group, we randomly selected 43 patients with MSS tumors treated in the CAIRO study as a validation group.

\section{Mitochondrial microsatellite instability (mtMSI) analysis}

Genomic DNA was extracted from four to eight manually microdissected 30- $\mu \mathrm{m}$ sections of FFPE tissue. Areas containing $>50 \%$ tumor cells were selected by microscopic evaluation on a reference slide stained with H\&E. Genomic DNA from microdissected tissues was isolated using the QIAamp ${ }^{\circledR}$ DNA micro kit (Qiagen, Valencia, CA) following the manufacturer's instructions. DNA concentration was determined at $260 \mathrm{~nm}$ using the Nanodrop ND-1000 spectrophotometer (Nanodrop Technologies, Inc., Wilmington, DE, USA). The sequence of mutational hotspot regions was determined by PCR and Sanger sequencing using six primer sets: D-loop $310\left(\mathrm{C}_{8} \mathrm{TC}_{6}\right)$, D-loop $514\left(\mathrm{CA}_{5}\right)$, D-loop $16184\left(\mathrm{C}_{12}\right)$, ND1 $\left(\mathrm{C}_{6}\right)$, ND5 $\left(\mathrm{C}_{6} \mathrm{~A}_{8}\right)$, and COX1 $\left(\mathrm{A}_{7}\right)$ (Supplemental Table 1) $[10,25,28,43]$. The fragments were analyzed for length variations in tumor tissue compared to normal tissue (instability). We read the electropherograms manually to identify variable sequence length present at low level and to determine if there was coexistence of different subpopulations of the mononucleotide tracts in tumor tissue and normal tissue (heteroplasmy). Heteroplasmy makes analysis with 
GeneScan, which is used for nuclear MSI, difficult. Some samples revealed a different proportion of mtDNA subpopulation between normal and tumor tissue. Such a difference can occur due to changes in the proportion of mtDNA subpopulations during mitochondrial segregation or due to mutations, and we also classified these as instable. The analyses were performed in duplicate on normal and tumor DNA of the selected patients. The results were independently scored by two observers. In case of inconsistent results, the entire mtMSI experiment was repeated, and the final conclusion was based on two similar results. Due to technical failure, only 152 instead of 182 patients were analyzed for ND5.

\section{Statistical analysis}

Patients were analyzed for the prevalence and prognostic value in four different groups: patients with combined MSI and mtMSI tumors (MSI/mtMSI), patients with combined MSI and mtMSS tumors (MSI/mtMSS), patients with combined MMS and mtMSI tumors (MSS/mtMSI), and patients with combined MSS and mtMSS tumors (MSS/mtMSS). The prevalence of mtMSI was compared for patients with MSI and MSS tumors. The outcome was analyzed within the group of patients with MSI tumors (excluding the Lynch syndrome patients) for mtMSI compared to mtMSS tumors and within the group of patients with MSS tumors for mtMSI compared to mtMSS tumors.

For the mtMSI analysis of the D-loop region (D310 locus, D514 locus, and D16184 locus) and per loci, patients were divided into two groups: stable versus instable tumors. The comparison of the prevalence of mtMSI between groups (MSI versus MSS) was performed by chi-square or Fisher's exact tests, where appropriate. OS was defined as the time from randomization until death from any cause. OS curves were estimated using the Kaplan-Meier method and compared using a Cox proportional hazard model. All tests were two-sided, and $p<0.05$ was considered as statistically significant. All analyses were conducted using the SAS system version 9.2.

Literature search strategy, inclusion criteria, and data extraction

We reviewed the literature on the prevalence of mtMSI in stage I-IV CRC patients. The primary outcome of interest was prevalence of mtMSI. A search was conducted of Medline, PubMed, and the Cochrane Library from January 1998 (year of the first publication of mtMSI in CRC) to January 2014 with an English-language restriction using the following search terms: mitochondrial microsatellite instability, mitochondrial mutations, and mitochondrial alterations, in combination with colon cancer and colorectal cancer. Original publications were selected if the abstract contained data for patients with mtMSI. In case of duplicate publications, the most recent and/or most complete study was included. We excluded publications of patients with colon cancer and $\mathrm{CRC}$ in which only mutations in the mitochondrial genome were described.

\section{Results}

Prevalence of mtMSI at the D-loop

mtMSI has been defined as change in length in short base repetitive sequences of mtDNA between normal and tumor tissue. This assumes that the normal colon tissue of CRC patients has a constant sequence pattern. This was checked for the D310 and D514 region in 10 patients from our hospital database for which we could obtain normal tissue from proximal and distal locations relative to the tumor. We analyzed the mtDNA with PCR and Sanger sequencing. In these 10 patients, we did not observe a difference in the normal tissues, but in five patients, the tumor did show an altered fragment length at the D310 region (Supplementary Table 2). This result confirms that mtMSI is a tumor-specific event and that the comparison of one tumor and one normal tissue block per patient should be sufficient to assess mtMSI. Figure 1 illustrates an example of mtMSI analyzed by direct sequencing. Analyses were done in duplicate. Overall, 54.4\% (99 out of 182) of all patients showed mtMSI at the D-loop region (D310 locus+D514 locus+D16184 locus). The majority of all patients showed mtMSI at the D310 locus $(50.5 \%)$, whereas instability at the D514 locus was found in $4.9 \%$ of all patients and instability at the D18164 locus in $1.6 \%$ of all patients (Fig. 2). mtMSI at the D-loop region was most often found at only one of the three D-loop loci (94 out of 99). Five patients showed instability at two loci, with the following combinations: D310 and D514 ( $n=2), \mathrm{D} 310$ and D16184 $(n=2)$, and D514 and D16184 $(n=1)$. Patients with MSI tumors showed slightly more often mtMSI at the D-loop region compared to patients with MSS tumors; 57.8 versus $51.5 \%, p=0.083$.

Prevalence of mtMSI at ND1, ND5, and COX1

Instability at the NADH dehydrogenase subunits ND1 and ND5 was rare. Only $1.1 \%$ of patients ( 2 out of 182 ) showed instability at the ND1 locus, and $4.6 \%$ (7 out of 153) of patients showed instability at the ND5 locus. None of the patients showed instability at the cytochrome oxidase subunit COX1.

Prognosis of mtMSI at the D-loop region

Baseline patient and tumor characteristics of all patients are summarized in Table 1. Survival data for mtMSI at the D-loop region (D310 locus+D514 locus+D16184 locus) are 

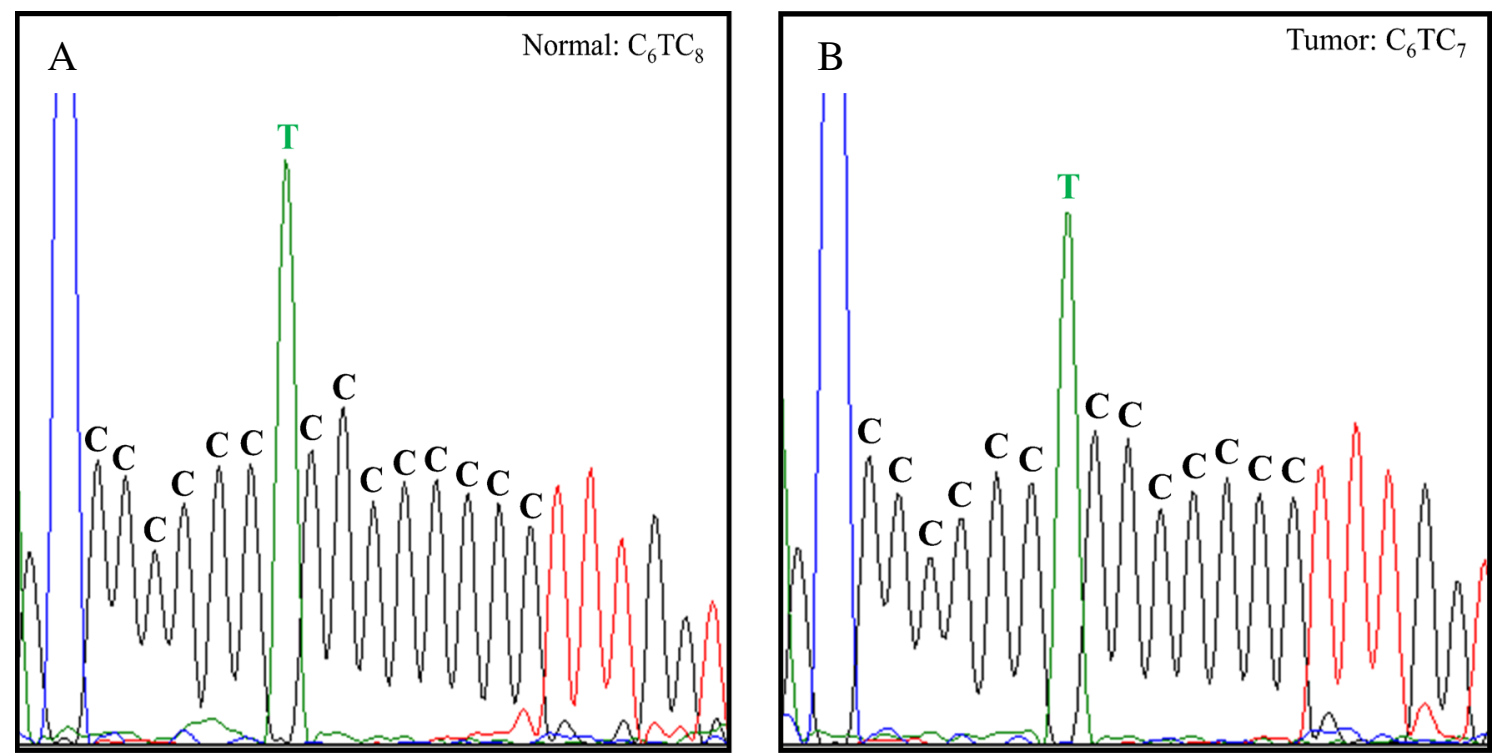

Fig. 1 Electropherograms obtained by direct sequencing of the mitochondrial D310 region of matched normal (a) and tumor $(\mathbf{b})$ tissue. The $(C)_{n}$ status of the major population differ in the samples. $\mathbf{a} \mathrm{C}_{6} \mathrm{TC}_{8}$ and $\mathbf{b} \mathrm{C}_{6} \mathrm{TC}_{7}$. This is an example of mtMSI

presented in Table 2. There was no correlation of OS with mtMSI at the D-loop region, when analyzing the three Dloop loci separately or together.

\section{Review of the literature}

The literature search identified 10 retrospective, non-randomized, single center studies in stage I-IV CRC (Fig. 3) [6, 11,
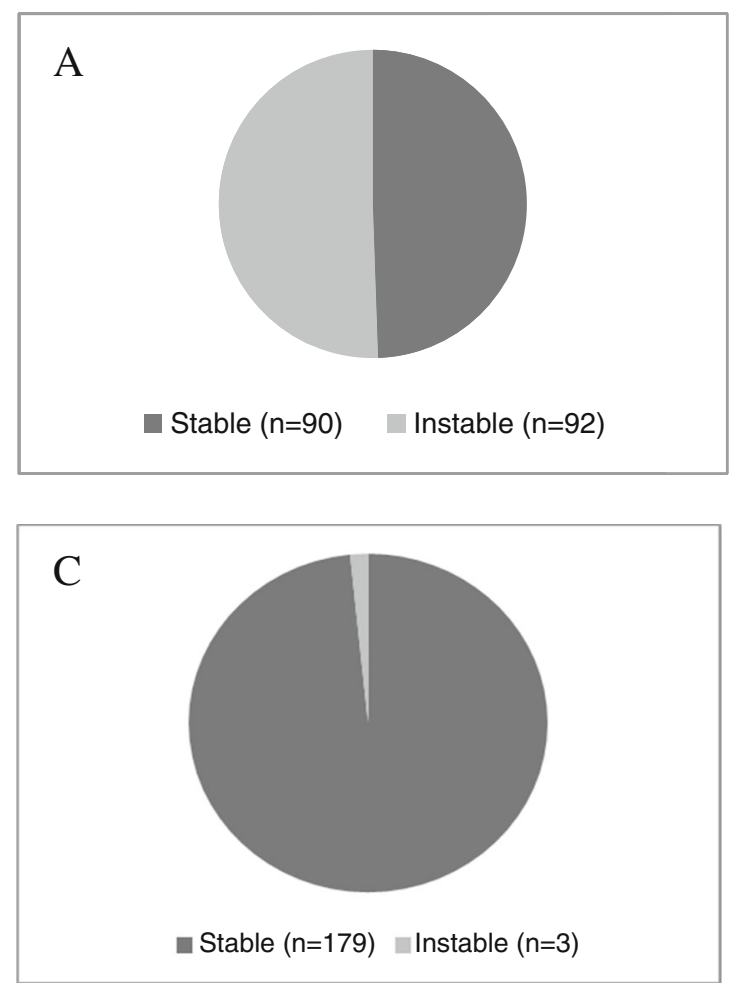

Fig. 2 Prevalence of mtMSI at the D310 locus (50.5\%) (a), at the D514 locus (4.9\%) (b), and at the D18164 locus (1.6\%) (c) of all patients

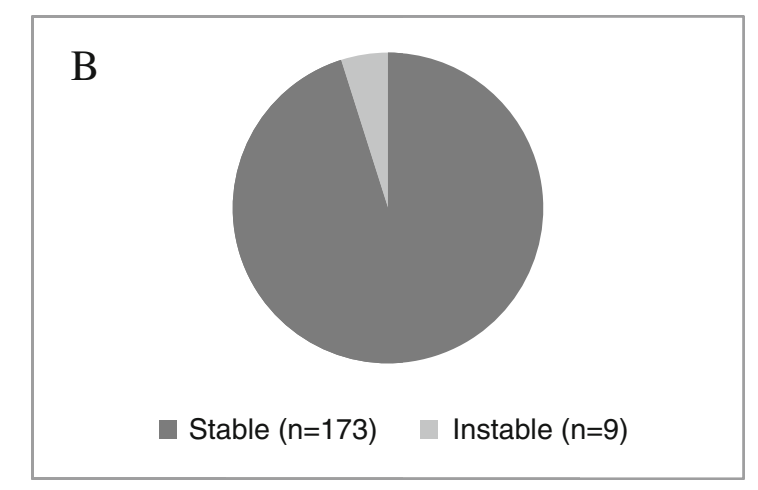

12, 24, 26-28, 34, 39, 43]. All studies analyzed the same loci and the same repeat; however, the definition of mtMSI was different among studies. Some studies used the same definition of mtMSI as we used in the current study [11, 12, 28, 39, 43]. While other studies describe insertion and deletions of $\mathrm{C}$ nucleotides in the mononucleotide repeat as mutations or alterations $[6,24,26,27,34]$. Two studies used band-shifting [12, 39], while all the other studies 
Table 1 Baseline patient and tumor characteristics of all patients treated in the CAIRO studies, subdivided by patients with MSI and MSS tumors

MSI microsatellite instability, MSS microsatellite stable

Table 2 Survival data of the patients with MSI and MSS tumors in relation to the mtMSI status of the D-loop region (D310 locus+D514 locus+D16184 locus)

$O S$ overall survival, $H R$ hazard ratio

\begin{tabular}{|c|c|c|c|}
\hline & \multirow{2}{*}{$\begin{array}{l}\text { Patients with MSI tumors } \\
(n=44)\end{array}$} & \multicolumn{2}{|c|}{ Patients with MSS tumors } \\
\hline & & $\begin{array}{l}\text { Test group } \\
(n=56)\end{array}$ & $\begin{array}{l}\text { Validation group } \\
(n=43)\end{array}$ \\
\hline Median age (range) & $68(34-78)$ & $66(34-79)$ & $68(39-81)$ \\
\hline \multicolumn{4}{|l|}{ Sex } \\
\hline Male & $24(55 \%)$ & $37(66 \%)$ & $28(65 \%)$ \\
\hline Female & $20(45 \%)$ & $19(34 \%)$ & $15(35 \%)$ \\
\hline \multicolumn{4}{|l|}{ WHO performance status } \\
\hline PS0 & $28(64 \%)$ & $56(100 \%)$ & $26(61 \%)$ \\
\hline PS1 & $14(32 \%)$ & - & $16(37 \%)$ \\
\hline PS2 & $2(4 \%)$ & - & $1(2 \%)$ \\
\hline \multicolumn{4}{|l|}{ Serum LDH } \\
\hline Normal & $32(73 \%)$ & $56(100 \%)$ & $29(67 \%)$ \\
\hline Abnormal & $12(27 \%)$ & - & $14(33 \%)$ \\
\hline \multicolumn{4}{|l|}{ Localization of the primary tumor } \\
\hline Colon & $38(86 \%)$ & $48(86 \%)$ & $26(61 \%)$ \\
\hline Recto sigmoid & $2(4 \%)$ & $8(14 \%)$ & $1(2 \%)$ \\
\hline Rectum & $3(7 \%)$ & - & $15(35 \%)$ \\
\hline Multiple tumor & $1(3 \%)$ & - & $1(2 \%)$ \\
\hline \multicolumn{4}{|l|}{ Metastatic sites involved } \\
\hline Median & 2 & 2 & 1 \\
\hline 1 & $16(36 \%)$ & $26(46 \%)$ & $22(51 \%)$ \\
\hline$>2$ & $26(60 \%)$ & $30(54 \%)$ & $21(49 \%)$ \\
\hline unknown & $2(4 \%)$ & - & - \\
\hline Predominant localization of metastases & - & & \\
\hline Liver & $14(32 \%)$ & $40(71 \%)$ & $27(63 \%)$ \\
\hline Liver + other & $13(30 \%)$ & - & - \\
\hline Extra-hepatic & $16(36 \%)$ & $15(27 \%)$ & $16(37 \%)$ \\
\hline Unknown & $1(2 \%)$ & $1(2 \%)$ & - \\
\hline \multicolumn{4}{|l|}{ Histology of the primary tumor } \\
\hline Mucinous (>50 \% WHO) & $16(36 \%)$ & $5(9 \%)$ & $4(9 \%)$ \\
\hline Adenocarcinoma with mucinous component & $4(9 \%)$ & $6(11 \%)$ & $4(9 \%)$ \\
\hline Adenocarcinoma & $23(52 \%)$ & $41(73 \%)$ & $34(80 \%)$ \\
\hline Unknown & $1(3 \%)$ & $4(7 \%)$ & $1(2 \%)$ \\
\hline
\end{tabular}

\begin{tabular}{|c|c|c|c|c|}
\hline & & & \multicolumn{2}{|l|}{ D-loop region } \\
\hline & & & mtMSS & mtMSI \\
\hline \multirow[t]{3}{*}{ MSI tumors } & & Number of patients & 17 & 27 \\
\hline & OS & Months $(95 \% \mathrm{CI})$ & $16.2(8.5-19.8)$ & $13.1(7.3-19.8)$ \\
\hline & & $\mathrm{HR}(95 \% \mathrm{CI})$ & $0.96(0.50-1.84)$ & \\
\hline \multirow{3}{*}{$\begin{array}{l}\text { Test group } \\
\text { (MSS tumors) }\end{array}$} & & Number of patients & 30 & 26 \\
\hline & OS & Months $(95 \% \mathrm{CI})$ & $19.1(17.1-28.6)$ & $20.1(13.6-26.2)$ \\
\hline & & HR $(95 \%$ CI $)$ & $1.20(0.67-2.12)$ & \\
\hline \multirow{3}{*}{$\begin{array}{r}\text { Validation group } \\
\text { (MSS tumors) }\end{array}$} & & Number of patients & 18 & 25 \\
\hline & OS & Months (95 \% CI) & $16.3(12.5-27.3)$ & $18.3(12.9-21.0)$ \\
\hline & & $\mathrm{HR}(95 \% \mathrm{CI})$ & $1.23(0.64-2.35)$ & \\
\hline
\end{tabular}


Fig. 3 Prevalence of mtMSI at the D310 locus at the D-loop in stage I-IV colorectal cancer patients among different studies
Prevalence of mtMSI in CRC among different studies

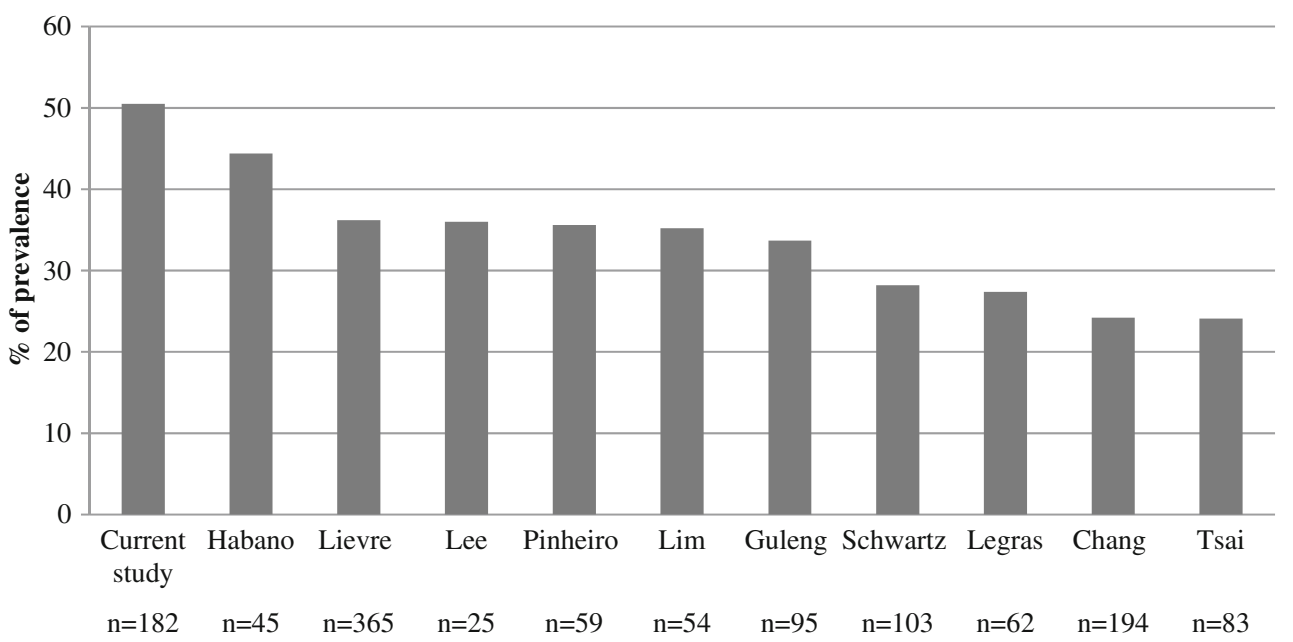

used direct sequencing to detect mtMSI. All studies evaluated the D310 locus separately. Prevalence of mtMSI at the D310 locus ranged from 24.1 to $44.4 \%$ (Fig. 3). Only one study evaluated the prevalence at other regions (D514 and D16184) of the D-loop, and they found mtMSI at the D16184 locus in $18.6 \%$ of patient and at the D514 locus in $7.4 \%$ of patients [28]. Prevalence of mtMSI at the ND1 and ND5 locus was low and ranged from 1.2 to $6.8 \%$ [12, 34, 43]. In Fig. 4, the different studies comparing the prevalence of mtMSI in stage I-IV CRC patients with MSI and MSS tumors are summarized in a forest plot $[11,27,34,39,43]$. mtMSI was not significantly more frequent in tumors with MSI (RR 1.07, $95 \%$ CI 0.88-1.30).

\section{Discussion}

In the current study, we demonstrate a high prevalence of mtMSI in mCRC patients with both MSI and MSS tumors. mtMSI was particularly present at the D310 locus. There was no correlation with prognosis. The D-loop is a triple stranded structure, and its attachment to the nuclear membrane with the subsequent exposure to lipid peroxides formed in the inner mitochondrial membrane makes this region prone for mutations in cancer cells (in particular the D310 region [29]). It is the most variable region in mtDNA and alterations, such as point mutations, insertions, deletions, and mtMSI are common events [3]. This is in line with the high prevalence of instability found at the D310 locus in the current study and previous published studies in stage I-IV CRC (Fig. 2a) [6, 11, 12, 24, 26-28, 34, 39, 43]. In our study, the prevalence of instability at the D-loop (and the D310 locus) is even higher compared to previous published studies [19, 24, 26-33]. The high prevalence might be caused by our selection of patients with metastatic disease, since some studies demonstrated a link between mtMSI with poor prognosis [27] and advanced stage of disease $[18,20]$. Alternatively, the variation of prevalence of instability at the D-loop might be caused by differences in the applied definitions and diagnostic panels. There are multiple potential pitfalls in the detection methodology [36]. Direct sequencing has a limited detection level and is hardly quantitative. The heteroplasmy makes it hard to determine whether an alteration results from genuine mutation or simply reflects changes in the proportion of mtDNA subpopulations during mitochondrial segregation. For future research, next generation sequencing and analyses of larger cohorts will be useful. Instability at the ND1 and ND5 genes, subunits of the NADH dehydrogenase
Fig. 4 Forest plot of the association of prevalence of mtMSI at the D310 locus in stage I-IV CRC patients with MSI compared to MSS tumors

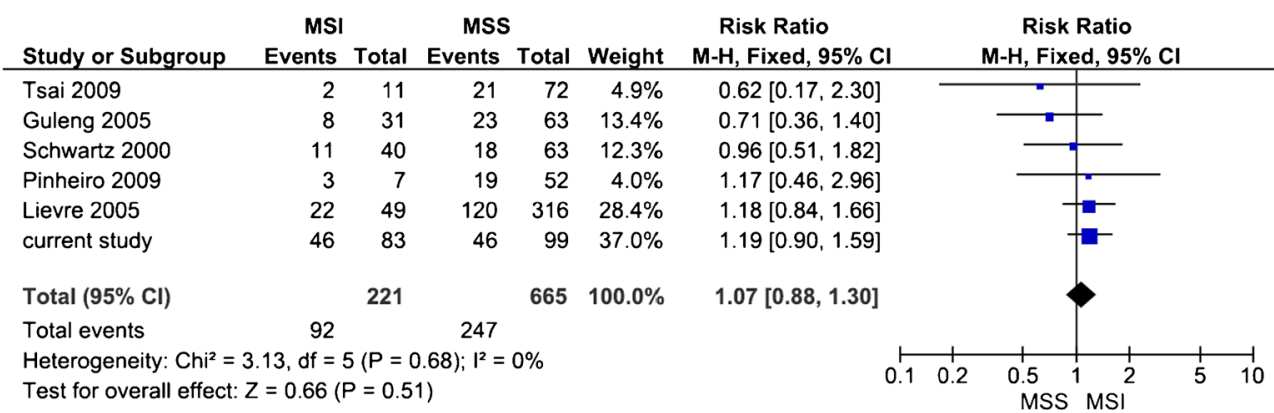


complex, is rare in CRC $[12,43]$ and only occurs in combination with instability at the D310 locus. This suggests that these might be secondary effects, similar to those observed in nuclear MSI [31].

The current study and previous studies on the correlation between nuclear MSI and mtMSI in CRC showed that nuclear MSI and mtMSI are independent events, suggesting that MMR enzymes are not involved in the correction of mtDNA base mispairing [11, 27, 34, 39, 43]. This is consistent with the fact that nuclear MMR proteins do not have any mitochondrial localization signal and cannot be detected in mitochondria by immunohistochemical analysis [13]. Furthermore, it was shown that in mitochondria, YB-1 mediates strong MMR activity, which is unrelated to nuclear MMR proteins [7, 32]. Important questions that still remain unanswered are the following: what are the functional consequences of mtMSI, is mtMSI a cause or a consequence of tumorigenesis, and especially, does mtMSI have prognostic value? The D-loop region is a crucial site for replication and expression of the mitochondrial genome because it contains essential transcription and replication elements [30, 45]. For that reason, one may speculate that sequence variations in this region can alter mtDNA replication and transcription and thus mitochondrial function. Then again, most of the variations are in the polymorphic length range and thus unlikely to lead to functional impairment of the mitochondria [38]. The prognostic value of mtMSI was only analyzed in four other studies in stage I-IV CRC patients, and results were inconsistent [6, 11, 27, 43]. One study found an association between alteration in the Dloop and poor prognosis in stage III CRC patients [27]. However, this was not confirmed in a subsequent study [43]. Also, in our study, there was no correlation with prognosis. Taken together, mtMSI at the D310 locus is frequent in CRC patients, but the prognostic value is still unclear.

Acknowledgments This study was supported by a grant of the Dutch Colorectal Cancer Group (DCCG).

\section{Conflict of interest None}

Funding This study was supported by the Dutch Colorectal Cancer Group (DCCG).

Open Access This article is distributed under the terms of the Creative Commons Attribution License which permits any use, distribution, and reproduction in any medium, provided the original author(s) and the source are credited.

\section{References}

1. Akouchekian M, Houshmand M, Akbari MH, Kamalidehghan B, Dehghan M (2011) Analysis of mitochondrial ND1 gene in human colorectal cancer. J Res Med Sci : Off J Isfahan Univ Med Sci 16:50-55
2. Akouchekian M, Houshmand M, Hemati S, Ansaripour M, Shafa M (2009) High rate of mutation in mitochondrial DNA displacement loop region in human colorectal cancer. Dis Colon Rectum 52:526530

3. Attardi G (1985) Animal mitochondrial DNA: an extreme example of genetic economy. Int Rev Cytol 93:93-145

4. Bianchi NO (2010) Mitochondrial genome instability in cancer. Cytogenet Genome Res 128:66-76

5. Carew JS, Huang P (2002) Mitochondrial defects in cancer. Mol Cancer 1:9

6. Chang SC, Lin PC, Yang SH, Wang HS, Liang WY, Lin JK (2009) Mitochondrial D-loop mutation is a common event in colorectal cancers with p53 mutations. Int J Colorectal Dis 24:623-628

7. de Souza-Pinto NC, Mason PA, Hashiguchi K, Weissman L, Tian J, Guay D, Lebel M, Stevnsner TV, Rasmussen LJ, Bohr VA (2009) Novel DNA mismatch-repair activity involving YB-1 in human mitochondria. DNA Repair (Amst) 8:704-719

8. DiMauro S, Schon EA (2003) Mitochondrial respiratory-chain diseases. N Engl J Med 348:2656-2668

9. Ericson NG, Kulawiec M, Vermulst M, Sheahan K, O'Sullivan J, Salk JJ, Bielas JH (2012) Decreased mitochondrial DNA mutagenesis in human colorectal cancer. PLoS Genet 8:e1002689

10. Gargano G, Calcara D, Corsale S, Agnese V, Intrivici C, Fulfaro F, Pantuso G, Cajozzo M, Morello V, Tomasino RM, Ottini L, Colucci G, Bazan V, Russo A (2007) (2007) Aberrant methylation within RUNX3 $\mathrm{CpG}$ island associated with the nuclear and mitochondrial microsatellite instability in sporadic gastric cancers. Results of a GOIM (Gruppo Oncologico dell'Italia Meridionale) prospective study. Ann Oncol 18(6):vi103-vi109

11. Guleng G, Lovig T, Meling GI, Andersen SN, Rognum TO (2005) Mitochondrial microsatellite instability in colorectal carcinomas-frequency and association with nuclear microsatellite instability. Cancer Lett 219:97-103

12. Habano W, Nakamura S, Sugai T (1998) Microsatellite instability in the mitochondrial DNA of colorectal carcinomas: evidence for mismatch repair systems in mitochondrial genome. Oncogene 17:19311937

13. Habano W, Sugai T, Nakamura SI, Uesugi N, Yoshida T, Sasou S (2000) Microsatellite instability and mutation of mitochondrial and nuclear DNA in gastric carcinoma. Gastroenterology 118:835-841

14. Habano W, Sugai T, Yoshida T, Nakamura S (1999) Mitochondrial gene mutation, but not large-scale deletion, is a feature of colorectal carcinomas with mitochondrial microsatellite instability. Int J Cancer 83:625-629

15. He Y, Wu J, Dressman DC, Iacobuzio-Donahue C, Markowitz SD, Velculescu VE, Diaz LA Jr, Kinzler KW, Vogelstein B, Papadopoulos $\mathrm{N}$ (2010) Heteroplasmic mitochondrial DNA mutations in normal and tumour cells. Nature 464:610-614

16. Heerdt BG, Chen J, Stewart LR, Augenlicht LH (1994) Polymorphisms, but lack of mutations or instability, in the promotor region of the mitochondrial genome in human colonic tumors. Cancer Res 54:3912-3915

17. Hibi K, Nakayama H, Yamazaki T, Takase T, Taguchi M, Kasai Y, Ito K, Akiyama S, Nakao A (2001) Detection of mitochondrial DNA alterations in primary tumors and corresponding serum of colorectal cancer patients. Int J Cancer 94:429-431

18. Ishikawa K, Takenaga K, Akimoto M, Koshikawa N, Yamaguchi A, Imanishi H, Nakada K, Honma Y, Hayashi J (2008) ROS-generating mitochondrial DNA mutations can regulate tumor cell metastasis. Science 320:661-664

19. Kassem AM, El-Guendy N, Tantawy M, Abdelhady H, El-Ghor A, Abdel Wahab AH (2011) Mutational hotspots in the mitochondrial D-loop region of cancerous and precancerous colorectal lesions in Egyptian patients. DNA Cell Biol 30:899-906 
20. Kim HS, Lim HS, Lee SH, Lee JW, Nam SW, Park WS, Lee YS, Lee JY, Yoo NJ (2006) Mitochondrial microsatellite instability of colorectal cancer stroma. Int J Cancer 119:2607-2611

21. Koopman M, Antonini NF, Douma J, Wals J, Honkoop AH, Erdkamp FL, de Jong RS, Rodenburg CJ, Vreugdenhil G, Loosveld OJ, van Bochove A, Sinnige HA, Creemers GJ, Tesselaar ME, Slee PH, Werter MJ, Mol L, Dalesio O, Punt CJ (2007) Sequential versus combination chemotherapy with capecitabine, irinotecan, and oxaliplatin in advanced colorectal cancer (CAIRO): a phase III randomised controlled trial. Lancet 370:135-142

22. Koopman M, Kortman GA, Mekenkamp L, Ligtenberg MJ, Hoogerbrugge N, Antonini NF, Punt CJ, van Krieken JH (2009) Deficient mismatch repair system in patients with sporadic advanced colorectal cancer. Br J Cancer 100:266-273

23. Larman TC, DePalma SR, Hadjipanayis AG, Cancer Genome Atlas Research N, Protopopov A, Zhang J, Gabriel SB, Chin L, Seidman CE, Kucherlapati R, Seidman JG (2012) Spectrum of somatic mitochondrial mutations in five cancers. Proc Natl Acad Sci U S A 109: 14087-14091

24. Lee HC, Yin PH, Lin JC, Wu CC, Chen CY, Wu CW, Chi CW, Tam TN, Wei YH (2005) Mitochondrial genome instability and mtDNA depletion in human cancers. Ann N Y Acad Sci 1042:109-122

25. Lee JH, Choi IJ, Song DK, Kim DK (2010) Genetic instability in the human lymphocyte exposed to hypoxia. Cancer Genet Cytogenet 196:83-88

26. Legras A, Lievre A, Bonaiti-Pellie C, Cottet V, Pariente A, Nalet B, Lafon J, Faivre J, Bonithon-Kopp C, Goasguen N, Penna C, Olschwang S, Laurent-Puig P (2008) Mitochondrial D310 mutations in colorectal adenomas: an early but not causative genetic event during colorectal carcinogenesis. Int J Cancer 122:2242-2248

27. Lievre A, Chapusot C, Bouvier AM, Zinzindohoue F, Piard F, Roignot P, Arnould L, Beaune P, Faivre J, Laurent-Puig P (2005) Clinical value of mitochondrial mutations in colorectal cancer. $\mathrm{J}$ Clin Oncol 23:3517-3525

28. Lim SW, Kim HR, Kim HY, Huh JW, Kim YJ, Shin JH, Suh SP, Ryang DW, Kim HR, Shin MG (2012) High-frequency minisatellite instability of the mitochondrial genome in colorectal cancer tissue associated with clinicopathological values. Int J Cancer 131:13321341

29. Mambo E, Gao X, Cohen Y, Guo Z, Talalay P, Sidransky D (2003) Electrophile and oxidant damage of mitochondrial DNA leading to rapid evolution of homoplasmic mutations. Proc Natl Acad Sci U S A 100:1838-1843

30. Marcelino LA, Thilly WG (1999) Mitochondrial mutagenesis in human cells and tissues. Mutat Res 434:177-203

31. Markowitz S, Wang J, Myeroff L, Parsons R, Sun L, Lutterbaugh J, Fan RS, Zborowska E, Kinzler KW, Vogelstein B et al (1995) Inactivation of the type II TGF-beta receptor in colon cancer cells with microsatellite instability. Science 268:1336-1338

32. Mason PA, Matheson EC, Hall AG, Lightowlers RN (2003) Mismatch repair activity in mammalian mitochondria. Nucleic Acids Res 31:1052-1058

33. Park SY, Shin MG, Kim HR, Oh JY, Kim SH, Shin JH, Cho YB, Suh SP, Ryang DW (2009) Alteration of mitochondrial DNA sequence and copy number in nasal polyp tissue. Mitochondrion 9:318-325
34. Pinheiro M, Veiga I, Pinto C, Afonso L, Sousa O, Fragoso M, Santos L, Lopes P, Pais I, Lopes C, Teixeira MR (2009) Mitochondrial genome alterations in rectal and sigmoid carcinomas. Cancer Lett 280: 38-43

35. Polyak K, Li Y, Zhu H, Lengauer C, Willson JK, Markowitz SD, Trush MA, Kinzler KW, Vogelstein B (1998) Somatic mutations of the mitochondrial genome in human colorectal tumors. Nat Genet 20: 291-293

36. Salas A, Yao YG, Macaulay V, Vega A, Carracedo A, Bandelt HJ (2005) A critical reassessment of the role of mitochondria in tumorigenesis. PLoS Med 2:e296

37. Sanchez-Cespedes M, Parrella P, Nomoto S, Cohen D, Xiao Y, Esteller M, Jeronimo C, Jordan RC, Nicol T, Koch WM, Schoenberg M, Mazzarelli P, Fazio VM, Sidransky D (2001) Identification of a mononucleotide repeat as a major target for mitochondrial DNA alterations in human tumors. Cancer Res 61:70157019

38. Schwartz S Jr, Alazzouzi H, Perucho M (2006) Mutational dynamics in human tumors confirm the neutral intrinsic instability of the mitochondrial D-loop poly-cytidine repeat. Genes Chromosomes Cancer 45:770-780

39. Schwartz S Jr, Perucho M (2000) Somatic mutations in mitochondrial DNA do not associate with nuclear microsatellite instability in gastrointestinal cancer. Gastroenterology 119:1806-1808

40. Shimomura T, Hiyama T, Oka S, Tanaka S, Yoshihara M, Shimamoto F, Chayama K (2011) Frequent somatic mutations of mitochondrial DNA in traditional serrated adenomas but not in sessile serrated adenomas of the colorectum. J Gastroenterol Hepatol 26:1565-1569

41. Shin MG, Kajigaya S, Tarnowka M, McCoy JP Jr, Levin BC, Young NS (2004) Mitochondrial DNA sequence heterogeneity in circulating normal human CD34 cells and granulocytes. Blood 103:4466-4477

42. Tol J, Koopman M, Cats A, Rodenburg CJ, Creemers GJ, Schrama JG, Erdkamp FL, Vos AH, van Groeningen CJ, Sinnige HA, Richel DJ, Voest EE, Dijkstra JR, Vink-Borger ME, Antonini NF, Mol L, van Krieken JH, Dalesio O, Punt CJ (2009) Chemotherapy, bevacizumab, and cetuximab in metastatic colorectal cancer. $\mathrm{N}$ Engl J Med 360:563-572

43. Tsai MH, Fang WH, Lin SW, Yen SJ, Chou SJ, Yang YC (2009) Mitochondrial genomic instability in colorectal cancer: no correlation to nuclear microsatellite instability and allelic deletion of hMSH2, hMLH1, and p53 genes, but prediction of better survival for Dukes' stage C disease. Ann Surg Oncol 16:2918-2925

44. Wallace DC (1992) Diseases of the mitochondrial DNA. Annu Rev Biochem 61:1175-1212

45. Wallace DC (1994) Mitochondrial DNA sequence variation in human evolution and disease. Proc Natl Acad Sci U S A 91:8739-8746

46. Wang CY, Li H, Hao XD, Liu J, Wang JX, Wang WZ, Kong QP, Zhang YP (2011) Uncovering the profile of somatic mtDNA mutations in Chinese colorectal cancer patients. PLoS One 6:e21613

47. Warburg O (1956) On the origin of cancer cells. Science 123:309314 